\title{
Comportamiento Reológico de Pulpas de Cuarzo a diferentes Concentraciones del Sólido
}

\author{
Adriana M. Osorio*, Juan M. Marín y Gloria Restrepo \\ Grupo Procesos Fisicoquímicos Aplicados, Departamento de Ingeniería Química, Facultad de Ingeniería, \\ Universidad de Antioquia UdeA; Calle 70 No. 52-21, Medellín, Colombia (e-mail: eamoc822@udea.edu.co) \\ ${ }^{*}$ Autor a quien debe ser dirigida la correspondencia
}

Recibido Jun. 18, 2014; Aceptado Ago. 19, 2014; Versión final recibida Sep. 29, 2014

\begin{abstract}
Resumen
El comportamiento viscoso de pulpas de cuarzo provenientes de moliendas fue caracterizado midiendo su viscosidad a diferentes tiempos de molienda y para varias concentraciones de sólidos, utilizando un viscosímetro Brookfield. Se usó el modificador reológico hidroxietilcelulosa (HEC) para preparar las suspensiones, evitar el problema de la sedimentación y evaluar la influencia de la concentración de sólidos en la pulpa y la diferencia en la granulometría. Los resultados indican que la característica newtoniana de una solución de HEC al $0.3 \%$ se ve afectado con el incremento de sólidos en la suspensión, siendo este efecto más marcado para una concentración de sólidos de $60 \%$. Se observó también que para una misma concentración de sólidos la disminución del parámetro de tamaño de la muestra no produce cambios significativos en el comportamiento reológico de la pulpa, para porcentajes bajos de sólidos (menor a 40\%).
\end{abstract}

Palabras clave: reología, viscosidad, molienda, cuarzo, newtoniano

\section{Rheological Behavior of Quartz Slurries at different Solid Concentrations}

\begin{abstract}
The viscous behavior of quartz pulp from mills was characterized by measuring their viscosities at different times for several grinding times and solid concentrations, using a Brookfield viscometer. The rheology modifier hydroxyethylcellulose (HEC) was used for preparing the suspensions, for avoiding the problem of sedimentation and for evaluating the influence of the concentration of solids on the pulp as well as on the difference in particle size. The results indicate that the Newtonian characteristic of a solution of $0.3 \% \mathrm{HEC}$ is affected by the increase of solids in the suspension. This effect is more notorious for a solid concentration of $60 \%$. It was also observed that for the same concentration, the solid size reduction parameter of the sample does not produce significant changes in the rheological behavior of the pulp, for low percentages (lower than $40 \%$ ).
\end{abstract}

Keywords: rheology, viscosity, grinding, quartz, Newtonian 


\section{INTRODUCCIÓN}

El comportamiento reológico de las pulpas minerales es indicativo del nivel de interacción o agregación entre partículas, de aquí deriva su importancia como variable de control en procesos tales como transporte de lodos, deshidratación y molienda húmeda (Muster and Prestidge, 1995), siendo esta última considerada de gran interés para la industria del procesamiento de minerales (Klimpel, 1982; Ding et al, 2007). Dentro de las moliendas industriales más usadas una de las más populares es la molienda con bolas, la cual consiste en introducir el material en un cilindro rotatorio y cargarlo con bolas para que se vaya reduciendo de tamaño por acción del movimiento de las bolas en el interior. Esta operación depende de condiciones operacionales tales como las dimensiones del molino, su velocidad de giro, la fracción de llenado y tamaño de bolas, el tamaño de partícula del alimento y del producto. Sin embargo a pesar de su importancia, en los últimos años la concentración de sólidos y la viscosidad de la pulpa han sido parámetros que han cobrado importancia al momento de determinar las condiciones de operación (Hiroto et al, 2004), puesto que al interior de una molienda en húmedo las propiedades físicas y químicas de la pulpa tales como la distribución y tamaño de partícula, la concentración de sólidos, el uso de dispersantes, el valor de $\mathrm{pH}$, la tasa de cizalla y la temperatura, cambian y por ende tienen una significativa influencia en la reología debido a que modifican sus propiedades fisicoquímicas superficiales (He et al, 2004).

Cuando una pulpa está compuesta por partículas muy finas (típico de una molienda fina), las partículas sólidas se depositarán muy lentamente y la agitación termal (movimiento Browniano) puede ser suficiente para mantener a las partículas suspendidas indefinidamente. La presencia de esos sólidos puede tener un efecto significante en las propiedades de la mezcla, resultando usualmente en un incremento agudo en la viscosidad de la mezcla comparado con la del líquido de transporte. Frecuentemente éstas mezclas exhiben comportamiento No Newtoniano (Gaitán, 2010). Casi todas las pulpas minerales industriales tienen un amplio rango de tamaños de partículas, frecuentemente abarcan varios órdenes de magnitud. Por lo tanto, el tamaño de las partículas minerales y concentración, usualmente cambiarán cuando se mueven a través de la planta o dentro de una unidad de operación (por ej. en un molino o en un espesador) (Gaitán, 2010).

En este sentido, se ha determinado que la adicción de partículas a un fluido Newtoniano da origen a una disminución de la energía mecánica que se disipa a medida que se incrementa la tasa de cizalladura, además la presencia de partículas finas puede dar paso a diversos fenómenos reológicos, tales como el cizallo adelgazante, cizallo espesante, formación de esfuerzo de fluencia y tixotropía entre otros (Willdemutth y Williams, 1985; Dabak y Yucel, 1987 y Bonifacio y Barrientos 2009), adicionalmente es importante señalar la existencia de un valor crítico en el espesamiento o concentración de sólidos, a partir del cual, el incremento de la viscosidad o del esfuerzo de fluencia se torna muy significativo (De Araujo et al, 2006). En una molienda estos comportamientos afectan marcadamente la eficiencia del proceso por tanto el estudio de dichas suspensiones requiere un conocimiento detallado de las múltiples variables que afectan su reología. El objetivo de este estudio fue determinar la influencia de variables como el tiempo de molienda, la concentración de sólidos y la variación de la granulometría en el comportamiento reológico de una pulpa del mineral cuarzo, producto de una molienda en un molino de bolas para determinar a futuro su impacto sobre la eficiencia de ésta.

\section{METODOLOGÍA}

\section{Equipos y accesorios}

Los principlaes equipos y accesorios usados en este trabajo son: Balanza Marcy para la determinación de la gravedad específica y preparación de las suspensiones; Serie de tamices Normalizada U.S.A Certificate Standard TestingSieve ASTM E 11/01 para la preparación de muestras y determinación de granulometrías; Viscosímetro Brookfield con accesorios para la determinación de la viscosidad; Molino de bolas; y Cuerpos moledores de acero al manganeso.

\section{Molienda de cuarzo}

Para la realización de las moliendas se seleccionaron muestras de un monotamaño de cuarzo, proveniente de la región, $-45+60$ mallas, $(355 \mu \mathrm{m}-250 \mu \mathrm{m})$. Y de acuerdo con las dimensiones del molino se seleccionaron y calcularon los parámetros operacionales, los cuales se presentan en la Tabla 1: Los tiempos de molienda fueron $1,3,5,7,10$ y 20 minutos.

\section{Preparación de las soluciones de HEC}

Se prepararon soluciones de HEC (hidroxietilcelulosa) grado comercial, a concentraciones de $0.1,0.2,0.3$, 0.5 y $1.0 \% \mathrm{w} / \mathrm{w}$, en agua destilada a $80^{\circ} \mathrm{C}$, a través de adición lenta del HEC bajo agitación constante, y se dejaron en reposo por un período de 24 horas, de acuerdo con el procedimiento descrito por Galván en 
1998 (Galván, 1998), para determinar hasta qué grado estas soluciones presentan un comportamiento newtoniano que permitiera su uso como solución blanco. Una vez identificadas las soluciones de carácter newtoniano y con el fin de realizar las mediciones reológicas, se seleccionaron aquellas que contribuían a minimizar el problema de la sedimentación y permitían dispersar las partículas de cuarzo de una forma adecuada.

Tabla 1: Condiciones de operación del molino

\begin{tabular}{|l|l|}
\hline Diámetro, $\mathrm{D}(\mathrm{m})$ & 0.155 \\
\hline Longitud, L(m) & 0.18 \\
\hline Fracción de llenado de bolas, J & 0.3 \\
\hline Velocidad crítica, Vc (RPM) & 124.4 \\
\hline Fracción de velocidad crítica, $\varphi \mathrm{c}$ & 0.75 \\
\hline Tamaño de bola (m) & 0.04 \\
\hline Fracción de intersticios entre bolas, U & 1.0 \\
\hline Fracción de llenado de polvo, fc & 1.12 \\
\hline Porcentaje de sólidos, \%V & 40 \\
\hline
\end{tabular}

\section{Calibración del viscosímetro}

Para la medición de viscosidad se seleccionaron dos fluidos con comportamiento newtoniano para la calibración del viscosímetro; en primer lugar se empleó agua destilada con el accesorio "UL adapter", el cual es adecuado para muestras de baja viscosidad y consiste en un eje cilíndrico que gira en el interior de una chaqueta cilíndrica. En segundo lugar se utilizó aceite de higuerilla grado técnico (proveedor local) para los diferentes rotores los cuales están diseñados para medir materiales viscosos.

\section{Preparación de las suspensiones polidispersas de cuarzo}

Una vez realizadas las moliendas en los tiempos seleccionados, se tamizaron los productos para así encontrar los parámetros de las distribuciones mediante un ajuste Rosin-Rammler (parámetro de distribución y parámetro de tamaño). Con las granulometrías obtenidas, se prepararon suspensiones de cuarzo al 20, 40 y $60 \%$ de sólidos para determinar como un cambio en el parámetro de tamaño de la distribución incide en el comportamiento de la viscosidad efectiva, $\mu_{\text {ef }}$ (viscosidad de la suspensión $\mu_{\mathrm{s}}$, respecto a la viscosidad del fluido sin adición de partículas, $\mu_{f}$ (Blissett y Rowson, 2013; Deosarkar y Sathe, 2012)) de la pulpa y para evaluar si a iguales parámetros de tamaño un cambio en la concentración de los sólidos afectan esa viscosidad efectiva.

\section{RESULTADOS Y DISCUSIÓN}

\section{Caracterización del material}

En la Tabla 2 se presentan algunas de las propiedades del material original: Para la realización de las moliendas se seleccionó un monotamaño de material correspondiente a la fracción -45+60 mallas, (355 $\mu \mathrm{m}$ $-250 \mu \mathrm{m})$.

Tabla 2: Características del material inicial de arena silícea (cuarzo)

\begin{tabular}{|l|l|}
\hline Tipo de material & Arena Silícea (cuarzo) \\
\hline$F_{80}(\mu \mathrm{m})$ & 4750 ( Malla 4) \\
\hline$P_{80}(\mu \mathrm{m})$ & 45 (Malla 325) \\
\hline Índice de trabajo, $\mathrm{W}(\mathrm{KWh} / \mathrm{tc})$ & 16 \\
\hline Densidad $\left(\mathrm{Kg} / \mathrm{m}^{3}\right)$ & 2.58 \\
\hline
\end{tabular}

Moliendas a diferentes intervalos de tiempo

Las distribuciones granulométricas producto de las moliendas a diferentes tiempos se ajustaron muy bien a una distribución Rosim-Rammler tal y como se aprecia en la Figura 1. 


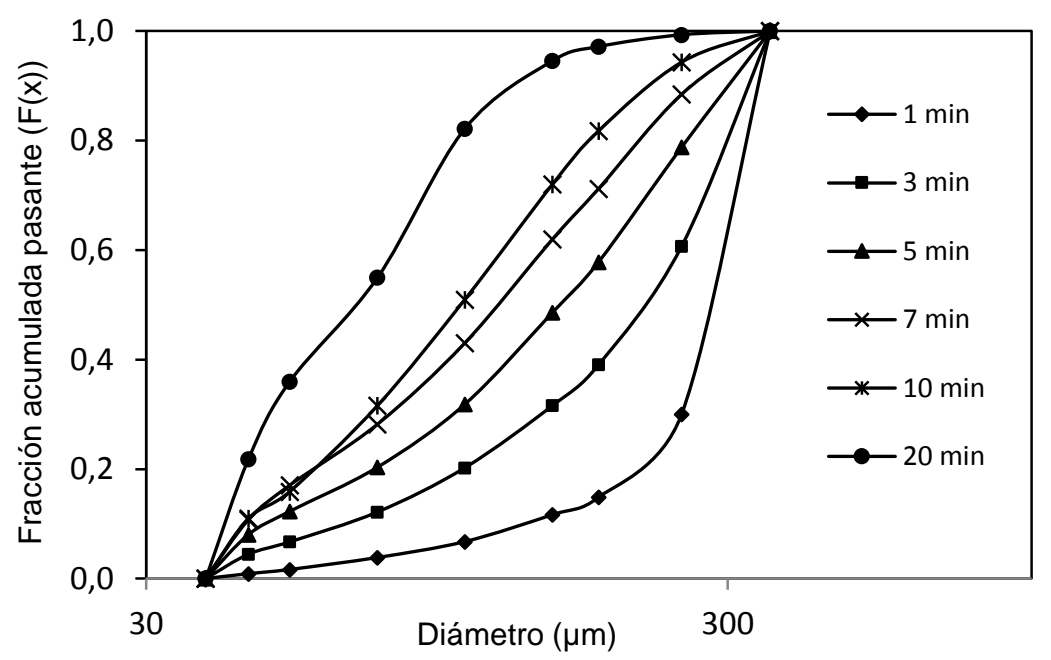

Fig. 1: Distribuciones granulométricas producto de las moliendas de la arena silícea (cuarzo)

Esta distribución se representa a través de la ecuación 1:

$F(x)=1-\left[-\left(\frac{x}{K_{R R}}\right)^{m}\right]$

En esta ecn., $F(x)$ es la fracción acumulada más fina que el tamaño $x, K_{R R}$ es el módulo de tamaño en micrones y $\mathrm{m}$ es el módulo de la distribución. La función de la ecuación 1 representa sólo algunas distribuciones de tamaño que son unimodales, esto es, que se puedan representar tan sólo por un parámetro de tamaño de la distribución $K_{R R}$ y un parámetro de distribución $m$. La importancia de esta ecuación radica en que muchos de los sistemas de partículas presentes en las corrientes usadas en equipos de la industria de procesamiento de sólidos, tal como la minera, pueden ser caracterizados por este tipo de distribución. Los parámetros que definen cada una de las distribuciones se presentan en la Tabla 3.

Tabla 3: Parámetros de los ajustes Rosim-Rammler para las distribuciones empleadas

\begin{tabular}{|c|c|c|c|}
\hline $\mathrm{t}$ de molienda $(\mathrm{min})$ & Par. tamaño, $\mathrm{K}_{\mathrm{RR}}$ & Par. distribución, $\mathrm{m}$ & $\mathrm{R}^{2}$ \\
\hline 1 & 447,25 & 1.88 & 0.9929 \\
\hline 3 & 262,40 & 1.69 & 0.9964 \\
\hline 5 & 191,93 & 1.63 & 0.9967 \\
\hline 7 & 154,07 & 1.64 & 0.9943 \\
\hline 10 & 134,39 & 1.85 & 0.9942 \\
\hline 20 & 83,06 & 1.74 & 0.9919 \\
\hline
\end{tabular}

\section{Calibración del viscosímetro}

Para la calibración del viscosímetro con los diferentes rotores se utilizó aceite de higuerilla, se realizaron mediciones por duplicado con lecturas ascendentes y descendentes. El rango permisible de error calculado de acuerdo con el procedimiento descrito en el manual del equipo para este caso fue entre $516-726 \mathrm{cp}$, la viscosidad medida fue de $717 \mathrm{cp}$ a $25{ }^{\circ} \mathrm{C}$ y el comportamiento exhibido fue el esperado, el de un fluido Newtoniano, semejante al presentado por Lozada y Velásquez en 2009 y los cuales reportaron una viscosidad de $621 \mathrm{cp}$ a 25 으 (Lozada y Velásquez, 2009). En el caso del accesorio UL Adapter, con el agua destilada, de igual forma se realizaron mediciones por duplicado con lecturas ascendentes y descendentes, el rango permisible de error calculado fue entre $0.53-1.51 \mathrm{cp}$, la viscosidad medida fue de $1.05 \mathrm{cp}$ a $25^{\circ} \mathrm{C}$, valor que resulta muy cercano al valor reportado por la literatura $\left(0.89 \mathrm{cp}\right.$ a $\left.25^{\circ} \mathrm{C}\right)$, la diferencia mínima tal vez se deba a las condiciones del laboratorio y de realización del ensayo, igualmente el comportamiento fue el de un fluido Newtoniano como se esperaba. Las curvas de fluidez se presentan en la Figura 2.

\section{Evaluación del comportamiento reológico de las soluciones de HEC}

Las soluciones de HEC al $0.1,0.2$ y $0.3 \% \mathrm{w} / \mathrm{w}$ exhibieron comportamientos newtonianos, las soluciones al 0.5 y $1.0 \%$ presentaron cierto grado de pseudoplasticidad. Al evaluar el proceso de sedimentación se seleccionó la solución al $0.3 \%(\mu \mathrm{f}=109 \mathrm{cp})$, debido a que permitía mantener las partículas suspendidas y dispersas para la realización de las mediciones reológicas en comparación con las de 0.1 y $0.2 \%$ las cuales presentaban precipitación rápida de partículas. La Figura 3 muestra este comportamiento. 

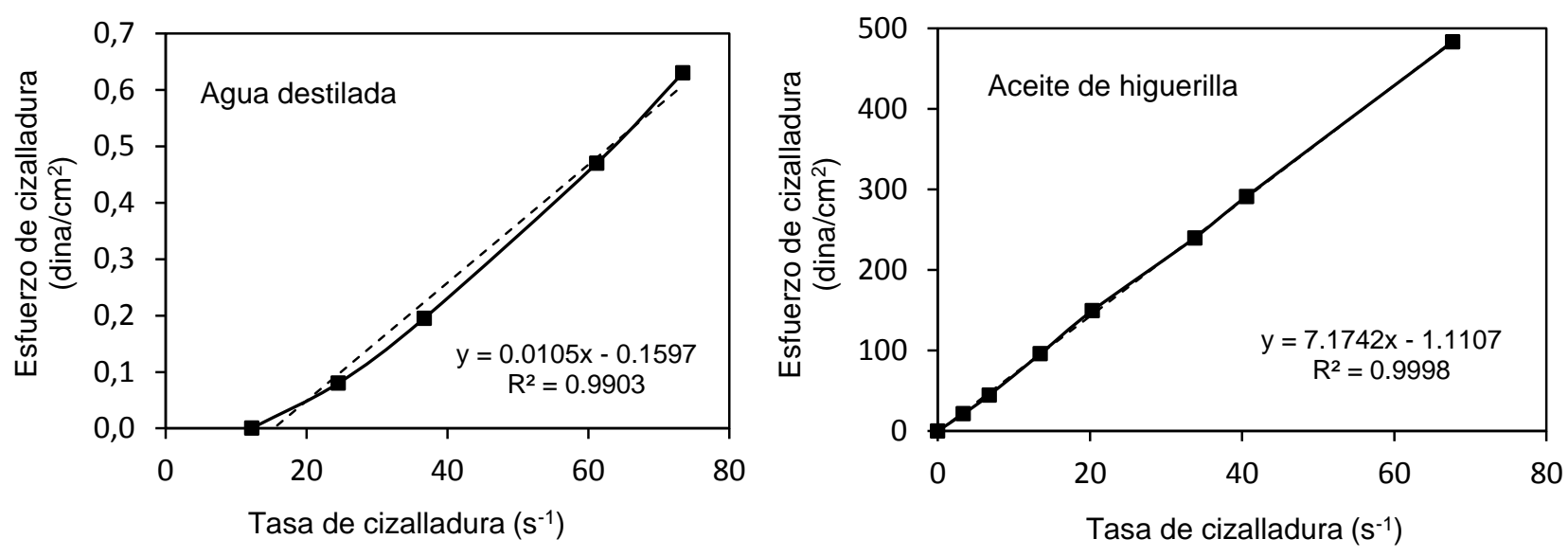

Fig. 2: Curvas de fluidez para los fluidos de calibración

\section{Evaluación del comportamiento de la viscosidad de la pulpa para diferentes tiempos de molienda}

Una vez obtenidas las granulometrías para los diferentes tiempos de molienda (Tabla 3), se prepararon muestras equivalentes en HEC al $0.3 \%$ (con igual porcentaje de sólidos al empleado en las moliendas y usando cada una de las granulometrías obtenidas para cada tiempo) con el fin de medir la viscosidad de la pulpa y evitar el problema de la sedimentación, de esta forma se pudo evaluar la relación de la viscosidad de la pulpa con la granulometría y el tiempo de molienda.

La Figura 4, presenta la evolución de la viscosidad de la pulpa con el tiempo de molienda y con el parámetro de tamaño. Es evidente que a mayor tiempo de molienda se obtiene una disminución del parámetro de tamaño $K_{R R}$ y con esto un aumento en la viscosidad efectiva de la suspensión.

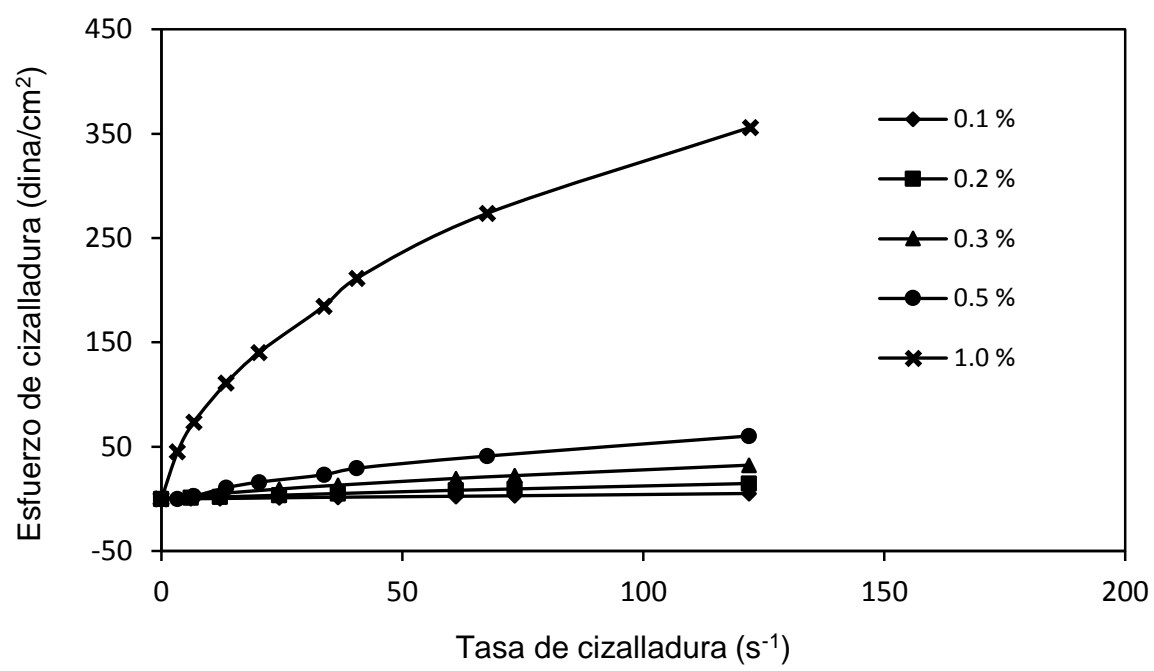

Fig. 3: Curva de fluidez para las soluciones de HEC

\section{Evaluación de la viscosidad de la pulpa}

Con las diversas granulometrías obtenidas bajo los diferentes tiempos de molienda se prepararon pulpas en diferentes concentraciones de sólidos, con el fin de evaluar como se ve afectada la reología de esa pulpa cuando se tiene un mismo parámetro de tamaño a diferentes concentraciones de sólidos en la suspensión. En la Figura 5 y la Figura 6 se presenta la variación de la viscosidad efectiva con la disminución en el parámetro $\mathrm{K}_{\mathrm{RR}}$, se observa que partículas con tamaños pequeños contribuyen mayormente a un aumento en la viscosidad. En cuanto a la concentración de sólidos puede observarse como la viscosidad efectiva crece considerablemente con el incremento en el porcentaje de sólidos (Senapati et al, 2010), sin embargo puede apreciarse que este efecto es más marcado para el porcentaje de sólidos del $60 \%$, en menor medida para el de $40 \%$ y casi nulo para el de $20 \%$, esto indica que efectivamente al aumentar el contenido de sólidos se presenta una disminución entre la distancia de las partículas y esto hace que su movimiento se vea restringido provocando un aumento en la viscosidad. La Figura 6 presenta las ecuaciones que representan la tendencia lineal del comportamiento descrito que permiten determinar la viscosidad en puntos no estimados. 

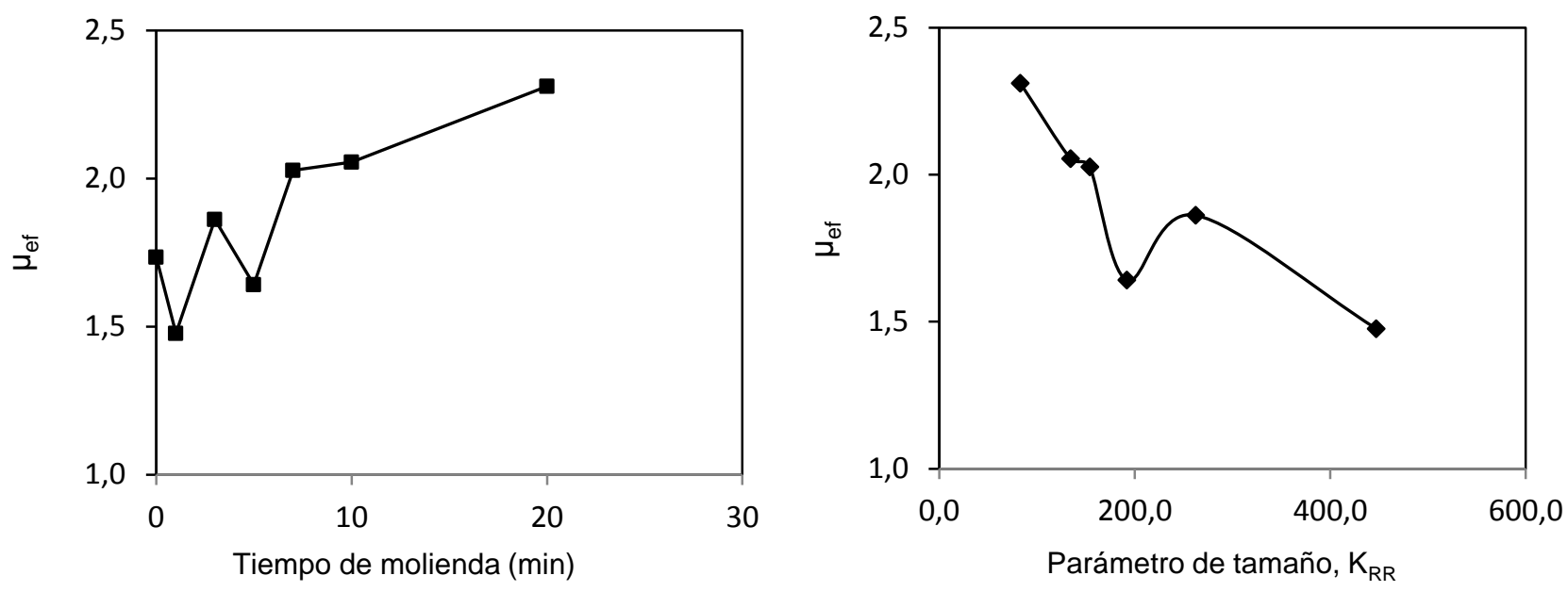

Fig. 4: Variación de la viscosidad efectiva con el tiempo y el parámetro $K_{R R}\left(J=0.3, \Phi_{c}=0.75, U=1.0\right)$

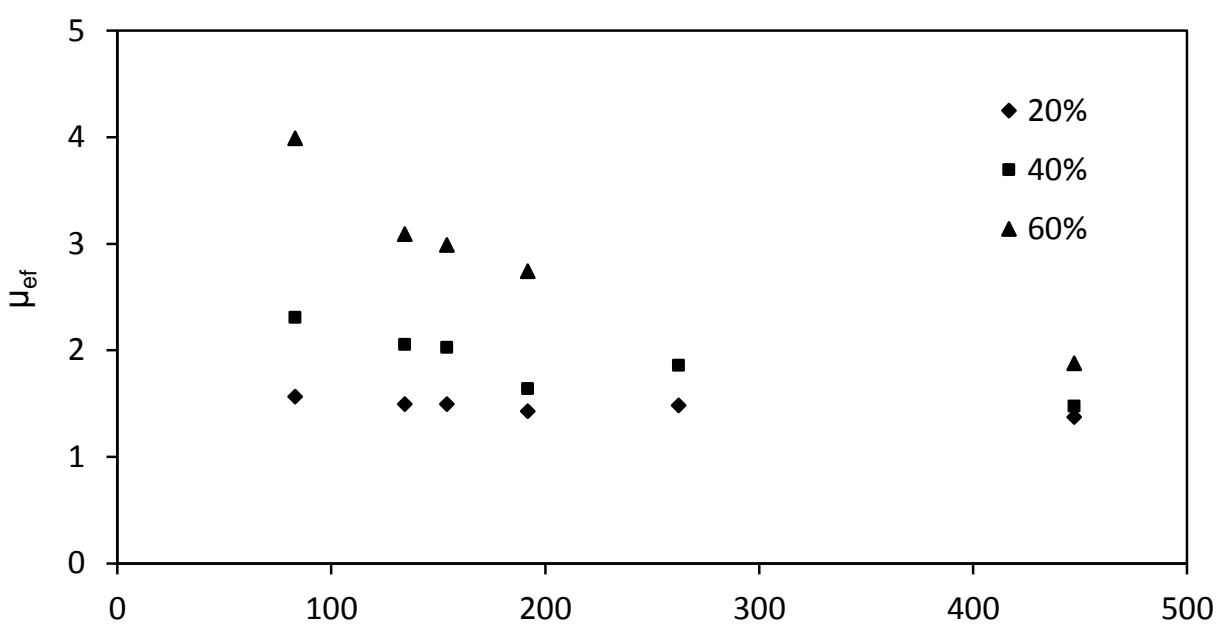

Parámetro de tamaño, $\mathrm{K}_{\mathrm{RR}}$

Fig. 5: Variación de la viscosidad efectiva con la concentración de sólidos

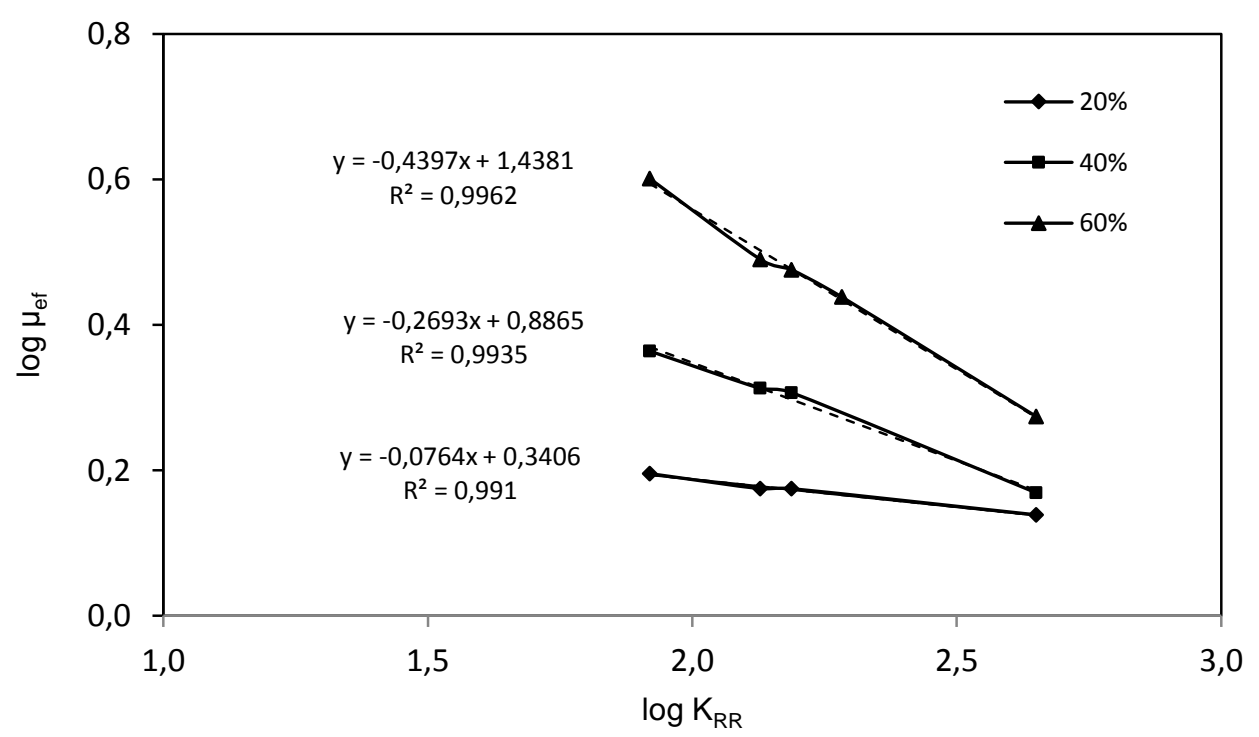

Fig. 6: Linealización de variación de la viscosidad efectiva con la concentración de sólidos 
Adicional a esto puede observarse que para los parámetros de tamaño mayores independiente del porcentaje de sólidos no se presentan cambios de viscosidad y esto indica que el incremento de la viscosidad posiblemente se da por el exceso de partículas relativamente finas que poseen escalas de movimiento comparativamente más grandes que los paquetes de partículas, y por ende modifican la reología del líquido que forma parte de la suspensión (Bustamante, 2002).

\section{CONCLUSIONES}

Se evaluó el comportamiento viscoso de pulpas de cuarzo provenientes de moliendas con molinos de bolas a diferentes tiempos de molienda, midiendo su viscosidad con un viscosímetro Brookfield, encontrándose que la viscosidad efectiva aumenta con la disminución en el parámetro $\mathrm{K}_{\mathrm{RR}}$ y con el incremento en la concentración de sólidos de la suspensión.

El incremento en la viscosidad efectiva tiene un efecto más marcado para el porcentaje de sólidos del $60 \%$, en menor medida para el de $40 \%$ y casi nulo para el de $20 \%$.

La soluciones de HEC presentaron comportamiento newtoniano en concentraciones de $0.1,0.2$ y $0.3 \%$, mientras las soluciones de $0.5 \%$ y de $1.0 \%$ exhibieron pseudoplasticidad. La solución de $0.3 \%$ de HEC resultó ser la apropiada para la preparación de las diferentes suspensiones de cuarzo.

El carácter newtoniano de la solución de $0.3 \%$ de HEC, se ve afectado por la adición de sólidos, cuyas interacciones entre partículas aumentan la resistencia. Con el aumento en la concentración de sólidos y la disminución del parámetro de tamaño $K_{R R}$ se ven afectadas las propiedades superficiales del sistema y por ende su comportamiento reológico.

\section{AGRADECIMIENTOS}

Los autores agradecen al programa Estrategia de Sostenibilidad 2011-2012 de la Universidad de Antioquia (Colombia) por el apoyo para el desarrollo de este trabajo.

\section{REFERENCIAS}

Blissett, R.S. y Rowson, N.A., An empirical model for the prediction of the viscosity of slurries of coal fly ash with varying concentration and shear rate at room temperature. Fuel. 111, 555-563, (2013).

Bonifacio, A. y Barrientos, A., Model for yield stress of quartz pulps and copper tailings. Int. J. Miner. Process. 93, 213-219, (2009).

Bustamante, O., Modelación matemática del tensor esfuerzo y de la viscosidad de una suspensión mineral. Tesis de Doctor en Ciencias Químicas, Dpto. Eng. Metalurgica, Universidad de Concepción, Concepción, Chile (2002).

Dabak, T. e Yucel, O., Modeling of the concentration and particle size distribution effects on the rheology of highly concentrated suspensions. Powder Technol. 52, 193-206, (1987).

De Araujo, A. y otros tres autores. Consistencia, Fluidez y Viscosidad de Pastas Minerales de Relaves de Hierro. Inf. tecnol. 17 (2), 71-79, (2006).

Ding, Z. y otros 3 autores. Effect of grinding parameters on the rheology of pyrite-heptane slurry in a laboratory stirred media mill. Miner. Eng. 20, 701-709, (2007).

Gaitán, I.P. Estimación de parámetros reológicos de pulpas minerales a diferentes concentraciones de sólidos. Tesis de Magíster en Ciencias con Mención en Ingeniería Hidráulica, Facultad de Ingeniería Civil, Universidad Nacional de Ingeniería, Lima, Perú (2010).

Galván, H., Reogramas de soluciones coloidales de metil celulosa y carboximetil celulosa sódica. Revista Colombiana de Ciencias Químico-Farmacéuticas. 27, 13-16, (1998).

He, M., Wang, Y. e Forssberg, E., Slurry rheology in wet ultra-fine grinding of industrial minerals: a review. Powder Technol. 147, 94-112, (2004).

He, M. y Forssberg, E. Influence of slurry rheology on stirred media milling of quartzite. Int. J. Miner. Process.8, 240-251, (2007). 
Hiroto, M., Hiroshi, M., Junya, K. y Fumio, S. Ball mill simulation in wet grinding using a tumbling mill and its correlation to grinding rate. Powder Technology 143-144, 230-239, (2004).

Kawatra, S.K., Bakshi, A.K. y Miller Jr. T.E. rheological characterization of mineral suspensions using a vibrating sphere and rotational viscosimeter. Int. J. Miner. Process.44-45, 155-165, (1996).

Klimpel, R.R., Slurry rheology influence on the performance of mineral/coal grinding circuits. Parts I and II.Min. Eng. 34 (12) 1665-1688, 35 (1), 21-26, (1982).

Deosarkar, M. y Sathe, V., Predicting effective viscosity of magnetite ore slurries by using artificial neural network. Powder Technology 219, 264-270, (2012).

Muster, T.H. y Prestidge, C.A. Rheological investigations of sulphide mineral slurries. Miner.Eng. 8, 15411555, (1995).

Senapati, P.K., Mishra, B.K. y Parida, A. Modeling of viscosity for power plant ash slurry at higher concentrations: Effect of solids volumen fraction, particle size and hydrodynamic interactions. Powder Technol. 197, 1-8, (2010).

Willdemutth, C.R. y Williams, M.C., A new interpretation of viscosity and yield stress in dense slurries: coal and irregular particles. Rheologica Acta 24, 75-91, (1985). 